\title{
EchoGéo
}

40 | 2017

Ressources urbaines (2)

\section{Dix ans !}

Jean-Louis Chaléard

\section{(2) OpenEdition}

Journals

Electronic version

URL: https://journals.openedition.org/echogeo/14987

DOI: 10.4000/echogeo.14987

ISSN: 1963-1197

\section{Publisher}

Pôle de recherche pour l'organisation et la diffusion de l'information géographique (CNRS UMR 8586)

\section{Electronic reference}

Jean-Louis Chaléard, “Dix ans !”, EchoGéo [Online], 40 | 2017, Online since 30 June 2017, connection on 10 August 2021. URL: http://journals.openedition.org/echogeo/14987 ; DOI: https://doi.org/10.4000/ echogeo. 14987

This text was automatically generated on 10 August 2021

EchoGéo est mis à disposition selon les termes de la licence Creative Commons Attribution - Pas d'Utilisation Commerciale - Pas de Modification 4.0 International (CC BY-NC-ND) 


\title{
Dix ans !
}

\author{
Jean-Louis Chaléard
}

1 Le quarantième numéro d'Échogéo marque la dixième année d'existence de la revue. En 2007, un petit groupe de chercheurs, enseignants-chercheurs et doctorants, pour beaucoup (mais pas tous!) appartenant à l'UMR Prodig se lançait dans l'aventure. Après bien des discussions et des hésitations, la revue naissait. Échogéo nous est apparu comme l'appellation la plus pertinente pour exprimer ce que nous voulions faire : une publication portant sur des thèmes de sociétés, d'environnement et de développement ; une information scientifique produite par des géographes mais aussi par d'autres spécialistes des sciences humaines et sociale, et destinée à un large public, qui dépasse les cercles universitaires. La formule d'une revue électronique allait de soi pour nous, en raison des facilités de diffusion et des coûts. Le dynamisme des premiers participants, le soutien de quelques grands noms de la géographie comme Paul Pélissier, l'appui de la plate-forme Revues.org contribuèrent à l'élargissement progressif de l'audience.

2 Depuis sa fondation, la revue a évolué, même si la maquette reste la même dans ses grandes lignes. Un des principaux fondateurs, rédacteur en chef durant 3 ans, Jean Marie Théodat, a dû quitter son poste, tout en restant fidèle au groupe. La rubrique Sur l'image est apparue en 2009. En 2011, les rubriques Sur le terrain et Sur le champ ont fusionné dans un souci de simplification, tandis que le varia a pris la place du dossier Sur le champ en fin d'année... L'élargissement du public a été de pair avec l'arrivée de collègues étrangers au comité de rédaction et la création d'un comité scientifique international en 2013. Notre succès doit beaucoup à la contribution de nombreux auteurs d'articles et à un lectorat actif et diversifié. Il repose aussi sur un système d'évaluation par des experts anonymes, soldats de l'ombre sans lesquelles la revue n'existerait pas dans sa dimension scientifique. Que tous soient ici remerciés, car si un petit groupe a eu l'initiative, ce sont bien les auteurs, les évaluateurs et les lecteurs qui font vivre la revue. Le format électronique nous a permis de toucher un public plus étendu que ne l'aurait fait une formule de revue sur papier, et c'est chaque fois avec plaisir que nous voyons le lectorat s'élargir géographiquement et de plus en plus de chercheurs d'autres continents, notamment d'Afrique, nous proposer des articles. 
3 Dès lors, il allait de soi que le thème de la table ronde organisée pour fêter notre anniversaire devait porter sur le numérique. Le 9 juin, des spécialistes se sont interrogés sur: "Le numérique: de nouvelles conditions du savoir?», abordant les vastes ressources autorisées par ce moyen de communication, les nouvelles formes de production et les formes de valorisation inédites. S'il est encore trop tôt pour rapporter sur cette table ronde, riche en débats, nous ne manquerons pas, dans un prochain numéro, d'en donner un compte-rendu.

4 La nouvelle livraison d'échogéo s'inscrit dans la continuité de ce que nous avons toujours essayé de faire, offrant des contributions dans chaque rubrique. Le dossier Sur le champ dirigé par A. Michel et A. Ribardière poursuit l'interrogation sur les «ressources urbaines » entamée dans le numéro 39. Deux thèmes avaient été déjà analysés : le premier portait sur la répartition des ressources dans la ville, le deuxième sur les pratiques de la ville par des acteurs au statut de citadin ou de citoyen peu consolidé ou précaire. Dans le présent numéro les deux derniers volets du dossier sont abordés reprenant et prolongeant des questions déjà évoquées. L'inégale répartition des ressources et des habitants dans la ville conduit à s'interroger sur les systèmes de mobilités, confrontant politiques publiques et pratiques des habitants. Les trois textes qui abordent la question, dans des contextes urbains très différents, mettent en évidence la forte pression sur les ressources du centre, les carences et/ou les dysfonctionnements des systèmes de transport qui handicapent en général les populations les plus démunies. Jean-Michel Kouakou Attien Konan souligne les difficulté de déplacement des habitants de Bouaké, deuxième ville de Côte d'Ivoire, à la suite de la crise politique des années 2000, l'apparition d'un nouveau mode de déplacement plus souple, mais aussi plus périlleux: les moto-taxis, et les comportements des conducteurs de taxi-automobiles, qui mettent en danger la vie des passagers. À Santiago du Chili, Claudia Arellano Yévenes et Fernando Saavedra Peláez montrent comment un même mode de transport, la bicyclette, recouvre des pratiques très différentes des habitants selon leur localisation dans la ville et leur catégorie sociale. Enfin, Solène Baffi analyse les résultats des politiques urbaines au Cap sur les transports et leurs effets. Après les pratiques ségrégationnistes du régime d'apartheid, puis les réorientations des politiques publiques depuis 1994 dans un cadre néolibéral, l'accessibilité réelle des populations laborieuses reste encore à construire, d'autant que les structures sociales et spatiales de la ville ont évolué, faisant apparaître de nouveaux besoins.

5 Les deux derniers articles insistent sur les ressources que les individus sont en capacité de mobiliser. Ils mettent en évidence l'agencement des ressources urbaines dans les modalités d'insertion des migrants d'origine rurale à Mexico. Antonine Ribardière, à partir de l'étude du district de Naucalpan, périphérie industrielle et résidentielle de la capitale mexicaine, relève que si la migration peut utiliser des relais familiaux, les trajectoires résidentielles et professionnelles ne s'inscrivent pas dans le cadre de filières strictes, et permettent de différencier les nouveaux migrants à la recherche d'emplois et de logements sur place, des migrants anciennement implantés qui s'affranchissent peu à peu du local. Aurélia Michel, s'appuyant sur des récits de vie, montre comment des populations dépourvues de ressources urbaines en acquièrent petit à petit en fonction des arbitrages et projets familiaux, privilégiant tantôt la centralité, tantôt les ressources de proximité. En conclusion, Monique Bertrand met en perspective les textes, à partir d'une analyse des travaux scientifiques antérieurs, 
soulignant les héritages, et mettant en évidence les filiations et les apports de la notion de "ressources urbaines ». Pour finir, elle suggère des approfondissements à partir de questions sur le terrain du "politique par le bas », ouvrant ainsi de nouveaux champs d'investigation à un thème déjà riche.

6 Les ressources urbaines sont également au cœur des trajectoires migratoires, c'est ce qu'aborde la rubrique Sur l'écrit. Le travail de Carolina Pinto-Baleisan auprès d'étudiants chiliens et colombiens à Paris et à New York montre que l'accès effectif aux ressources dans des contextes migratoires et universitaires tendus et compétitifs passe avant tout par l'activation des liens sociaux. Le capital social peut tour à tour donner lieu à des ressources "encapsulées", ou au contraire ouvrir à toutes les bifurcations biographiques.

7 L'intérêt d'une revue numérique est d'autoriser un large recours à l'image, que ce soit des cartes, des photographies, des documents divers. La rubrique Sur l'image est constituée cette fois-ci de six contributions qui complètent et illustrent abondamment les articles sur les ressources urbaines, qu'elles approfondissent un volet du dossier ou qu'elles proposent un autre point de vue. Ainsi, tour à tour, sont présentées des images sur les nouveaux bus du Cap, l'approvisionnement en eau d'une favela de Rio, le patrimoine bâti à Ho Chi Minh-ville, les ateliers de confection à Sao Paulo, les marchés foncier et immobilier à Mexico, l'accès aux ressources urbaines dans un espace non étudié dans le dossier : les villes mexicaines de la frontière nord, avec les États-Unis.

La rubrique Sur le métier aborde une question qui met en jeu la représentation visuelle et la collecte des données. Félix Lebfevre, Emmanuel Bonnet et Florence Boyer exposent une méthode de cartographie participative réalisée auprès d'habitants de Ouagadougou, sur la base de "focus groups ». L'intérêt du travail est de permettre d'atteindre une compréhension fine des pratiques et des représentations de la ville par ses habitants.

Dans la rubrique Sur le vif, l'article de Emmanuel Grégoire et Laurent Gagnol porte sur l'orpaillage, activité dite souvent «informelle », qui connaît un grand développement en Afrique subsaharienne. Les auteurs montrent comment la soif de l'or s'est emparée de nombreux Nigériens et étrangers en quête de fortune à la suite de la découverte de gisements d'or dans le nord du Niger. Ils abordent aussi les relations entre l'État, les commerçants et les orpailleurs et en analysent les conséquences à partir de l'étude de la région d'Agadez. L'exploitation de l'or a stabilisé politiquement la région, freiné les migrations vers d'autres pays et affaibli les djihadistes qui « ne peuvent plus s'appuyer sur la pauvreté ». Les leçons de l'article donnent ainsi à réfléchir sur des questions bien plus vastes que celle de l'orpaillage. 